\title{
Brane World Solution to the Cosmological Constant Problem
}

\author{
S.-H. Henry Tye* and Ira Wasserman ${ }^{\dagger}$ \\ Laboratory for Nuclear Studies and Center for Radiophysics and Space Research, Cornell University, Ithaca, New York 14853
} (Received 9 June 2000)

\begin{abstract}
We consider a model with two parallel (positive tension) 3-branes separated by a distance $L$ in fivedimensional spacetime. If the interbrane space is anti-de Sitter, or is not precisely anti-de Sitter but contains no event horizons, the effective four-dimensional cosmological constant seen by observers on one of the branes (chosen to be the visible brane) becomes exponentially small as $L$ grows large.
\end{abstract}

DOI: $10.1103 /$ PhysRevLett.86.1682

Recent observational data [1] indicate that there is a positive cosmological constant in the universe, which, compared to the Planck or the electroweak scale, is many orders of magnitude smaller than expected within the context of ordinary gravity and quantum field theory. This is the well-known cosmological constant problem [2]. Here we propose a solution to this problem in which the cosmological constant becomes exponentially small compared to all the other scales in the model.

The scenario is a variant of the Randall-Sundrum model [3]. Let us consider two parallel 3-branes (or two stacks of 3-branes) in 5D spacetime separated by a distance $L$ in the fifth dimension, which is not necessarily compactified. Let the brane on the left be the visible brane, with positive brane tension $\sigma_{0}$. The brane on the right has positive brane tension $\sigma_{L} \neq \sigma_{0}$, generally. Assume that the bulks outside the branes are anti-de Sitter (AdS) spaces, with cosmological constants $-\Lambda_{l}$ and $-\Lambda_{r}$, respectively. There may be numerous solutions of the 5D Einstein equations for the behavior of the bulk between the branes, but let us focus attention on the simplest, an AdS space with bulk cosmological constant $-\Lambda_{m}$. (We discuss other possibilities briefly below, and in more detail elsewhere.) We shall see that $\Lambda_{m}$ may be expressed as a function of $L, \Lambda_{l}$, $\Lambda_{r}, \sigma_{0}, \sigma_{L}$, and the 5D gravitational coupling constant $\kappa^{2}=8 \pi G$. Thus, we regard $\Lambda_{m}$ as a derived parameter; more generally, in a dynamical 5D spacetime, $\Lambda_{m}$ may evolve with time as branes move together or apart. For this simplest model for the interbrane spacetime, we can determine the effective 4D cosmological constant $\Lambda_{\text {eff }}$ in terms of $\Lambda_{l}, \Lambda_{r}, \sigma_{0}, \sigma_{L}, L$, and $\kappa^{2}$. We show that for large $L$,

$$
\Lambda_{\mathrm{eff}} \approx F\left(\kappa, \Lambda_{l}, \Lambda_{r}, \sigma_{0}, \sigma_{L}\right) e^{-2 \alpha_{0} L},
$$

where $F$ is independent of $L$ and the positivity of the 4D Newton constant $G_{N}$ requires $\alpha_{0}=\kappa^{2} \sigma_{0} / 3-$ $\sqrt{\kappa^{2} \Lambda_{l} / 6}>0$. For $\alpha_{0} L \gg 1$, the effective 4D cosmological constant becomes exponentially small in this simple model without any fine tuning of the parameters. In a $S^{1} / Z_{2}$ orbifold version of the model, the only bulk is between the branes, and

$$
\Lambda_{\text {eff }} \approx 2 \sigma_{0} \frac{\left(\sigma_{L}+\sigma_{0}\right)}{\left(\sigma_{L}-\sigma_{0}\right)} e^{-\kappa^{2} \sigma_{0} L / 3} .
$$

PACS numbers: $04.50 .+\mathrm{h}, 11.10 . \mathrm{Kk}, 98.80 . \mathrm{Cq}, 98.80 . \mathrm{Hw}$

These results remain true even if $\sigma_{0}$, which is simply the vacuum energy density of the 4D quantum field theory (which includes the standard model of strong and electroweak interactions) on the visible brane, changes due to phase transitions or other dynamics on the brane. We shall also comment briefly on solutions where the bulk between branes is not a pure AdS space. Demanding the absence of an event horizon between the branes, we again find $\Lambda_{\text {eff }}$ to be vanishing small for large $L$. This suggests that an exponentially small effective cosmological constant may be a robust property of two-brane models with large interbrane separation. One is tempted to speculate that the pure AdS solution is a stable fixed point as the branes move apart and the 3-brane universe expands.

When $\alpha_{0} L$ is not large, $\Lambda_{\text {eff }}(L)$ is more complicated than Eqs. (1) and (2). Furthermore, at small separations, we expect additional nongravitational brane-brane interactions, but for large separations, it is reasonable to assume that the brane dynamics is dominated by pure gravity as described here. In a more realistic situation, the matter density on the visible brane (and dark matter on the other brane) should be included, and the separation distance $L$ should be treated as a dynamical variable. For slowmoving branes, a Born-Oppenheimer-like approach is valid, and the spacetime evolves quasistatically from one nearly time-independent solution to another along the sequence of two-brane models. The result resembles the quintessence picture [4] and will be discussed elsewhere. Below, we derive Eqs. (1) and (2) from solutions with time-independent expansion rate and brane separation, i.e., in the quasistatic approximation.

Consider a pair of 3-branes at $y=0$ and $y=L$ with different positive brane tensions $\sigma_{0}$ and $\sigma_{L}$. Let the bulk cosmological constant be $-\Lambda_{l}$ for $y<0,-\Lambda_{r}$ for $y>L$. Choose the energy-momentum tensor for the bulk between the branes to have the diagonal form $T_{A B}=$ $\left(-\lambda_{0}, \lambda, \lambda, \lambda, \psi\right)$, where $\lambda_{0}(y), \lambda(y)$, and $\psi(y)$ are functions of $y$. Choosing $\lambda_{0}=\lambda$ allows us to use the metric ansatz

$$
d s^{2}=d y^{2}+A(y)\left[-d t^{2}+e^{2 H t} \delta_{i j} d x^{i} d x^{j}\right] .
$$

The $G_{05}$ component of the Einstein's equation $G_{A B}=$ $\kappa^{2} T_{A B}=8 \pi G T_{A B}$ is satisfied trivially, while the $G_{00}$ and the $G_{55}$ components give, respectively, 


$$
\begin{aligned}
\frac{A^{\prime \prime}}{A} & =\frac{2 H^{2}}{A}+\frac{2 \kappa^{2}}{3}\left[\Lambda_{l} \Theta(-y)+\lambda(y) \Theta(y) \Theta(L-y)+\Lambda_{r} \Theta(y-L)-\sigma_{0} \delta(y)-\sigma_{L} \delta(y-L)\right], \\
\left(\frac{A^{\prime}}{A}\right)^{2} & =\frac{4 H^{2}}{A}+\frac{2 \kappa^{2}}{3}\left[\Lambda_{l} \Theta(-y)+\psi(y) \Theta(y) \Theta(L-y)+\Lambda_{r} \Theta(y-L)\right] .
\end{aligned}
$$

The $G_{i j}$ component does not yield additional equations (alternatively, with the above metric, this component requires $\lambda_{0}=\lambda$ ). In the bulk between the branes, we have [differentiate $\psi(y)$ in Eq. (4) and manipulate]

$$
\psi^{\prime}+\frac{2 A^{\prime}}{A}(\psi-\lambda)=0
$$

It is clear that if $\psi=\lambda$ then $\lambda(y)=\psi(y)=\Lambda_{m}$, independent of $y$.

Let us first consider this particularly simple case, in which the space between the branes $(0<y<L)$ is AdS, with a cosmological constant $-\Lambda_{m}$ that will be determined from the solution of the 5D Einstein equations. Define $k_{l}=\sqrt{\kappa^{2} \Lambda_{l} / 6}, \quad k_{m}=\sqrt{\kappa^{2} \Lambda_{m} / 6}$, and $k_{r}=\sqrt{\kappa^{2} \Lambda_{r} / 6}$. Outside the branes, the solution for the bulks is

$$
\begin{aligned}
& A(y)=\frac{H^{2} \sinh ^{2}\left[k_{l}\left(y+y_{l}\right)\right]}{k_{l}^{2}}(y<0), \\
& A(y)=\frac{H^{2} \sinh ^{2}\left[k_{m}\left(y+y_{m}\right)\right]}{k_{m}^{2}}(0<y<L), \\
& A(y)=\frac{H^{2} \sinh ^{2}\left[k_{r}\left(y+y_{r}\right)\right]}{k_{r}^{2}}(y>L),
\end{aligned}
$$

where $y_{l}, y_{m}$, and $y_{r}$ are constants. These bulk solutions are similar to those in Ref. [5]. Continuity of the metric at the branes implies that

$$
\begin{aligned}
\frac{\sinh ^{2}\left(k_{l} y_{l}\right)}{k_{l}^{2}} & =\frac{\sinh ^{2}\left(k_{m} y_{m}\right)}{k_{m}^{2}}, \\
\frac{\sinh ^{2}\left[k_{m}\left(L+y_{m}\right)\right]}{k_{m}^{2}} & =\frac{\sinh ^{2}\left[k_{r}\left(L+y_{r}\right)\right]}{k_{r}^{2}} .
\end{aligned}
$$

The jump conditions at the two branes are

$$
\begin{array}{r}
\frac{k_{l} \cosh \left(k_{l} y_{l}\right)}{\sinh \left(k_{l} y_{l}\right)}-\frac{k_{m} \cosh \left(k_{m} y_{m}\right)}{\sinh \left(k_{m} y_{m}\right)}=q_{0}, \\
\frac{k_{m} \cosh \left[k_{m}\left(L+y_{m}\right)\right]}{\sinh \left[k_{m}\left(L+y_{m}\right)\right]}-\frac{k_{r} \cosh \left[k_{r}\left(L+y_{r}\right)\right]}{\sinh \left[k_{r}\left(L+y_{r}\right)\right]}=q_{L} .
\end{array}
$$

where $q_{0} \equiv \kappa^{2} \sigma_{0} / 3$ and $q_{L} \equiv \kappa^{2} \sigma_{L} / 3$.

$$
\begin{aligned}
k_{m} L & =\sinh ^{-1}\left(\frac{k_{m}}{H(0)}\right)+\sinh ^{-1}\left(\frac{k_{m}}{H(L)}\right) \\
& =\sinh ^{-1}\left(\frac{2 k_{m} q_{0}}{\sqrt{\left[k_{m}^{2}-\left(k_{l}+q_{0}\right)^{2}\right]\left[k_{m}^{2}-\left(k_{l}-q_{0}\right)^{2}\right]}}\right)+\sinh ^{-1}\left(\frac{2 k_{m} q_{L}}{\sqrt{\left[k_{m}^{2}-\left(k_{r}+q_{L}\right)^{2}\right]\left[k_{m}^{2}-\left(k_{r}-q_{L}\right)^{2}\right]}}\right) .
\end{aligned}
$$

Here, Eq. (12) is regarded as a relation that determines $k_{m}$ in terms of $L, q_{0}, q_{L}, k_{l}$, and $k_{r}$. In general, $H(0) \neq$ $H(L)$. Because of the condition (11), $H \rightarrow 0$ as $k_{m} \rightarrow$ $\alpha_{0}=q_{0}-k_{l}$ from below. This means that the expansion rate as seen by observers on the visible brane becomes exponentially small for large $L$,
The expansion rate seen by observers on the brane at $y=0$ is $H(0)=H / \sqrt{A(0)}$, where

$$
\begin{aligned}
\frac{H^{2}}{A(0)} & =\frac{k_{l}^{2}}{\sinh ^{2}\left(k_{l} y_{l}\right)}=\frac{k_{m}^{2}}{\sinh ^{2}\left(k_{m} y_{m}\right)} \\
& =\frac{\left[k_{m}^{2}-\left(k_{l}+q_{0}\right)^{2}\right]\left[k_{m}^{2}-\left(k_{l}-q_{0}\right)^{2}\right]}{4 q_{0}^{2}},
\end{aligned}
$$

with $k_{m}^{2}-\left(k_{l} \pm q_{0}\right)^{2}>0$ or $<0$, in agreement with Ref. [6], which uses a slightly different approach. Similarly, the expansion rate seen by observers on the brane at $y=L$ is $H(L)=H / \sqrt{A(L)}$, where

$$
\begin{aligned}
\frac{H^{2}}{A(L)} & =\frac{k_{r}^{2}}{\sinh ^{2}\left[k_{r}\left(L+y_{r}\right)\right]}=\frac{k_{m}^{2}}{\sinh ^{2}\left[k_{m}\left(L+y_{m}\right)\right]} \\
& =\frac{\left[k_{m}^{2}-\left(k_{r}+q_{L}\right)^{2}\right]\left[k_{m}^{2}-\left(k_{r}-q_{L}\right)^{2}\right]}{4 q_{L}^{2}},
\end{aligned}
$$

with $k_{m}^{2}-\left(k_{r} \pm q_{L}\right)^{2}>0$ or $<0$. We can rescale $t$ so that $A(0)=1$, and the Hubble constants on the two branes are, respectively, $H(0)=H$ and $H(L)=H / \sqrt{A(L)}$. Note that although Eqs. (9) and (10) appear to determine the expansion rates on the two branes completely in terms of local quantities (i.e., the local brane tensions, and bulk cosmological constants just outside each brane), the values of these quantities on/near the two branes are connected via $k_{m}$ and $y_{m}$.

The 4D Newton's constant $G_{N}$ can be determined by introducing a small matter density $\rho$ to the visible brane, that is, $q_{0} \rightarrow q_{0}+\kappa^{2} \rho / 3$. Requiring the Hubble constant $H$ to have the standard form $H^{2} \approx 8 \pi G_{N}\left(\Lambda_{\text {eff }}+\rho+\right.$ $\ldots) / 3$ yields $4 \pi q_{0} G_{N}=\kappa^{2} \alpha_{0} k_{l}$ [6-9]. Positivity of $G_{N}$ requires $\alpha_{0}=q_{0}-k_{l}>0$; to be specific, let us consider

$$
0 \leq k_{m} \leq q_{0}-k_{l} \text {. }
$$

Since the expansion rates $H$ and $H(L)$ given in Eqs. (9) and (10) depend on $k_{m}$, our goal is to express $H$ and $H(L)$ as functions of $L$ and the parameters $k_{l}, k_{r}, q_{0}$, and $q_{L}$. This requires an expression relating $L$ and $k_{m}$; from Eqs. (7) and (8) we find 
given by Eq. (10) and so does $C$. This implies that $\Lambda_{\text {eff }}$ becomes exponentially small as $L$ increases.

In the symmetric case, where $k_{l}=k_{r}=k$ and $q_{0}=$ $q_{L}=q$ (and let $\left.\alpha=q-k=\alpha_{0}\right)$, we have $H(L)=$ $H(0)=H$, and $y_{m}=-y_{l}=y_{r}=-L / 2$. The constant $C$ in Eq. (13) becomes $C=\alpha L / 2$, so, including a matter density $\rho$ (which is treated as a perturbation),

$$
H^{2} \approx 4 \alpha^{2} e^{-\alpha L}+\frac{2 \kappa^{2} \alpha k}{3 q} \rho .
$$

Thus, $\Lambda_{\text {eff }}$ still decreases exponentially with $L$, but slower than in the nonsymmetric case.

To this point, we have concentrated on spacetimes that are noncompact in $y$, but similar results can be derived for the compactified case. First, we may choose to identify $k_{l}=k_{r}=k_{m}$ and derive $k_{m}$ and $H$ in terms of $L$. Next, we can compactify the $y$ direction and further perform a $Z_{2}$ orbifold, with one brane sitting at each of the two fixed points $(y=0, L)$. This $S^{1} / Z_{2}$ orbifold model is particularly simple, since there is only one bulk space between the branes sitting at the two end points. This is an expanding (nonsupersymmetric) version of the HoravaWitten model [10], with branes with tension $\sigma_{0}=3 q_{0} / \kappa^{2}$ at $y=0$ and $\sigma_{L}=3 q_{L} / \kappa^{2}$ at $y=L$, separated by AdS space with $\Lambda_{m}=-6 k^{2} / \kappa^{2}$. The solution is

$$
A(y)=\frac{H^{2}}{k^{2}} \sinh ^{2}\left[k\left(y-s y_{0}\right)\right],
$$

where $s=+1$ for $y>0$ and $s=-1$ for $y<0$. Because of the orbifold symmetry of the model, we need to consider only the jump conditions at $y=0$ and $y=L$, which are $2 k / q_{0}=\tanh k y_{0}$ and $2 k / q_{L}=\tanh \left[k\left(L-y_{0}\right)\right]$, respectively. Combining the jump conditions implies

$$
\frac{q_{0}}{2 k}=\frac{\tanh k L-q_{L} / 2 k}{1-\left(q_{L} / 2 k\right) \tanh k L} ;
$$

if $q_{L} / 2 k= \pm 1$, then $q_{0} / 2 k=\mp 1$ irrespective of $k L$, but for $q_{L} / 2 k \neq 1, q_{0} / 2 k \rightarrow 1$ as $k L \rightarrow \infty$. According to our viewpoint, Eq. (16) determines $k$ given $q_{0}, q_{L}$, and $L$.

The expansion rates on the branes are $H(0)=H / \sqrt{A(0)}$ and $H(L)=H / \sqrt{A(L)}$, where $H^{2}(0)=k^{2}\left[\left(q_{0} / 2 k\right)^{2}-\right.$ 1] and $H^{2}(L)=k^{2}\left[\left(q_{L} / 2 k\right)^{2}-1\right]$. For large values of $q_{0} L$ we find that

$$
H^{2}(0) \approx \frac{q_{0}^{2}\left(q_{L}+q_{0}\right)}{\left(q_{L}-q_{0}\right)} e^{-q_{0} L} .
$$

Thus, for $q_{0} L \gg 1$ and $\left|q_{L}\right|>q_{0}>0$, the cosmological constant on the $y=0$ brane is positive, and exponentially small. Moreover, although Eq. (17) may appear singular as $q_{L} \rightarrow q_{0}$, in fact $H^{2}(0) \approx q_{0}^{2} e^{-q_{0} L / 2}$ in that case. If $q_{L}=-q_{0}$, as considered in Ref. [3], then $y_{0}= \pm \infty$, so $H(0)=H(L)=0$ and therefore $\left|q_{0}\right|=2 k$ for any finite nonzero $L$. Our model is qualitatively different, since it involves two branes with tensions of different magnitudes and not necessarily opposite signs, and interprets the bulk cosmological constant as a parameter derived from the brane tensions and separation. However, unlike the model of Ref. [3], ours does not solve the mass hierarchy problem, because $A(0) \gg A(L)$, but more complicated variants (e.g., with $>2$ branes) might.

Notice that $A(y)$ given by Eq. (6) may vanish at certain values of $y$. We view points where $A(y)=0$ as horizons. (There are no spacetime singularities at these points, however.) We do not worry about horizons outside the branes $(y<0$ and $y>L$ ), which recede to large distances from the branes as $L$ increases, but a horizon between the branes might be worrisome. To avoid a horizon between branes, we need $y_{m}<L+y_{m}<0$, which can be true if $k_{r}^{2}-q_{L}^{2}-\alpha_{0}^{2}>0$. This condition can be satisfied when the two branes and the outside bulks are not identical, but in the symmetric case, avoiding a horizon between the branes requires $\alpha<0$, which violates the positivity of $G_{N}$. So, in the symmetric case, we will have a horizon right between the branes even when they are close together $(L \rightarrow 0)$. (Nongravitational interbrane interaction could alter this conclusion for very small $L$, but not in general.) In the orbifold case, although we can allow arbitrary values of $q_{0}$ and $q_{L}$, horizons are avoided in the model only if $L<y_{0}$, which implies $q_{L}<0$. Horizons can also be avoided if the space between the branes is not pure AdS.

There is nothing sacred about AdS space between the branes (see [11]), and we might expect non-AdS behavior to be the rule rather than the exception, particularly at different stages in the evolution of a slowly changing 5D spacetime. Generally speaking, we can find infinitely many families of solutions with different $\lambda(y)$ and $\psi(y)$ in the regions between branes. As a first stab at a more complicated two-brane model than we have presented here, we have studied the case where $\lambda=\Lambda_{m}$ is a constant, but $\psi(y)=\Lambda_{m}-K / A^{2}(y)$, which satisfies Eq. (5) for $y$-independent $K$. In this case, we do not get a relation between $k_{m}$ and $L$ [because $\psi(y) \neq \Lambda_{m}$ ]. The question of whether $H^{2}$ is small is related to horizon formation between the branes. If horizons are absent, then $H^{2}$ must be exponentially small for large $L$. If a horizon may appear only between branes at large $L$, then $H^{2}$ must also be exponentially small. In a dynamical solution with separating branes, we might imagine that $H^{2} \rightarrow 0$ as $t \rightarrow \infty$, and no horizon forms.

To be specific, let us consider the orbifold model in some detail [12]. For $K>0$ and $A(0) \equiv 1$,

$$
A(y)=-\frac{H^{2}}{2 k^{2}}+\left(1+\frac{H^{2}}{2 k^{2}}\right) \frac{\cosh \left[2 k\left(y-s y_{0}\right)\right]}{\cosh \left(2 k y_{0}\right)},
$$

with $s$ chosen as for AdS. In this case the jump conditions at $y=0$ and $y=L$ are 


$$
\begin{aligned}
& \frac{q_{0}}{2 k}=\left(1+\frac{H^{2}}{2 k^{2}}\right) \tanh \left(2 k y_{0}\right), \\
& \frac{q_{L}}{2 k}=\left(1+\frac{H^{2}}{2 k^{2}}\right) \frac{\sinh \left[2 k\left(L-y_{0}\right)\right]}{A(L) \cosh \left(2 k y_{0}\right)}
\end{aligned}
$$

using the first of these relations in the second implies

$$
\begin{aligned}
\frac{H^{2}}{k} & =\frac{\left[\left(q_{0}+q_{L}\right)\right] \cosh (2 k L)-\left(2 k+q_{0} q_{L}\right) \sinh (2 k L)}{\sinh (2 k L)+\left(q_{L} / 2 k\right)[1-\cosh (2 k L)]} \\
& \approx\left(q_{0}-2 k\right)\left(1+\frac{2 q_{L} e^{-2 k L}}{q_{L}-2 k}\right) .
\end{aligned}
$$

Horizons are guaranteed to be absent in this model if

$$
\frac{H^{2}}{2 k^{2}}<\frac{1}{\cosh \left(2 k y_{0}\right)-1} \approx 2 e^{-2 k y_{0}} .
$$

Using Eq. (20) in the jump condition at $y=0$ implies $e^{-2 k y_{0}} \approx e^{-k L} \sqrt{q_{L}\left(q_{0}-2 k\right) / q_{0}\left(q_{L}-2 k\right)}$, and so horizons are absent if $q_{0} / 2 k-1<4 q_{L} e^{-2 k L} /\left(q_{L}-2 k\right)$ in the general case, $q_{L} \neq q_{0}$. If $q_{0}=q_{L}$, then $e^{-2 k y_{0}} \approx$ $e^{-k L}$ and horizons are avoided if $q_{0} / 2 k-1<2 e^{-k L}$. In either case, the expansion rate is exponentially small if there are no horizons in the solution.

The dynamical treatment of such a separation modulus may follow that discussed in Ref. [13]; conditions under which there are acceptable cosmological solutions are being investigated. At small separations, we expect brane-brane interactions beyond pure gravity. For large separations, it is reasonable to assume that the brane-brane interaction is dominated by pure gravity as described above. In Ref. [8] where an AdS-Schwarzschild solution is considered, the deviation from the pure AdS space redshifts away rapidly as the universe expands. This leads one to conjecture that the pure AdS solution between the branes is a stable fixed point. If so, as branes move apart and the universe expands, the bulk solution remains horizonfree, and approaches pure AdS space asymptotically. Clearly this issue needs careful investigation. It remains to be seen if fine-tuning is required for successful dynamical cosmological solutions.

One may even envision a multibrane scenario, where the separation distances between branes play the roles of various scalar fields, as suggested by string/ $M$ theory. In particular, the separation of two nearby branes may play the role of an inflaton [14], while the separation distance between two far-apart branes may play the role of a scalar field similar to quintessence [4]. Immediately after inflation, the separation of two nearby branes is stabilized by short-range brane-brane interactions [13,14], when the effective cosmological constant is small compared to the radiation density. Then a third brane starts to move away while the 3-brane universe is expanding, yielding an exponentially small $\Lambda_{\text {eff }}$.

We thank Eanna Flanagan and Horace Stoica for useful discussions. This research is partially supported by NSF (S.-H.H. T.) and NASA (I. W.).

*Electronic address: tye@mail.lns.cornell.edu ${ }^{\dagger}$ Electronic address: ira@spacenet.tn.cornell.edu

[1] A. Balbi et al., astro-ph/0005124 (MAXIMA-1); A.E. Lange et al., astro-ph/0005004 (BOOMERANG); A.G. Riess et al., Astron. J. 116, 1009 (1998); S. Perlmutter et al., Astrophys. J. 517, 565 (1999); See also N. A. Bahcall, J. P. Ostriker, S. Perlmutter, and P. J. Steinhardt, Science 284, 1481 (1999).

[2] For some useful reviews, see, e.g., S. Weinberg, Rev. Mod. Phys. 61, 1 (1989); S. M. Carroll, W. H. Press, and E. L. Turner, Annu. Rev. Astron. Astrophys. 30, 499 (1992); S. Weinberg, astro-ph/0005265; E. Witten, hep-ph/0002297; S. M. Carroll, astro-ph/0004075.

[3] L. Randall and R. Sundrum, Phys. Rev. Lett. 83, 3370 (1999); 83, 4690 (1999).

[4] B. Ratra and P. J. E. Peebles, Phys. Rev. D 37, 3406 (1988); C. Wetterich, Nucl. Phys. B302, 668 (1988); I. Zlatev, L. Wang, and P. J. Steinhardt, Phys. Rev. Lett. 82, 896 (1999).

[5] N. Kaloper, Phys. Rev. D 60, 123506 (1999); T. Nihei, Phys. Lett. B 465, 81 (1999); H. B. Kim and H. D. Kim, Phys. Rev. D 61, 064003 (2000).

[6] H. Stoica, S.-H.H. Tye, and I. Wasserman, Phys. Lett. B 482, 205 (2000).

[7] C. Csaki, M. Graesser, C. Kolda, and J. Terning, Phys. Lett. B 462, 34 (1999); J. M. Cline, C. Grojean, and G. Servant, Phys. Rev. Lett. 83, 4245 (1999); T. Shiromizu, K. Maeda, and M. Sasaki, Phys. Rev. D 62, 024012 (2000).

[8] P. Kraus, J. High Energy Phys. 9912, 011 (1999); E. E. Flanagan, S.-H.H. Tye, and I. Wasserman, Phys. Rev. D 62, 044039 (2000); P. Binétruy, C. Deffayet, U. Ellwanger, and D. Langlois, Phys. Lett. B 477, 285 (2000).

[9] More precisely, $4 \pi G_{N} q_{0}=\kappa^{2} \alpha_{0} k_{l}\left[1+2 \kappa^{2} \Lambda_{\text {eff }}\left(2 \alpha_{0}+\right.\right.$ $\left.\left.k_{l}\right) / 3 q_{0} k_{l}\right]$. Although $L$-dependent, the correction is small if $G_{N} \Lambda_{\text {eff }} / k_{l}^{2} \ll 1$, which is the case here.

[10] P. Horava and E. Witten, Nucl. Phys. B460, 506 (1996); B475, 94 (1996).

[11] O. DeWolfe, D. Z. Freedman, S. S. Gubser, and A. Karch, hep-th/9909134; P. Kanti, I. I. Kogan, K. A. Olive, and M. Pospelov, Phys. Lett. B 468, 31 (1999); C. Csaki, M. Graesser, L. Randall, and J. Terning, Phys. Rev. D 62, 045015 (2000).

[12] P. Kanti, K. A. Olive, and M. Pospelov, hep-ph/0005146.

[13] W. D. Goldberger and M. B. Wise, Phys. Rev. Lett. 83, 4922 (1999); Phys. Lett. B 475, 275 (2000); H. A. Chamblin and H. S. Reall, Nucl. Phys. B562, 133 (1999).

[14] G. Dvali and S.-H. H. Tye, Phys. Lett. B 450, 72 (1999); E. E. Flanagan, S.-H. H. Tye, and I. Wasserman, Phys. Rev. D 62, 024011 (2000). 